$$
|\alpha| \leqq\left|1-\frac{1}{2} \mu\right|+\frac{1}{2} \sum_{n=2}^{\infty} M^{n} /(2 n-1) !
$$

Hence, by the first of the inequalities (23),

$$
|\alpha|<\left|1-\frac{1}{2} \mu\right|+2-\frac{1}{2} \mu \text {. }
$$

On the other hand, the second of the inequalities (23) can be written in the form $\mu \geqq 2$, which means that

$$
\left|1-\frac{1}{2} \mu\right|=\frac{1}{2} \mu-1 \text {. }
$$

This completes the proof, since the last two formula lines imply the inequality $|\alpha|<1$, which is (9).

Conclusion. If $\mu, M$ are defined by (10), (11), then either (12) or (23) [and so, in particular, either (13) or (24)] is sufficient for stability.

As an illustration, let

$$
f(t)=(a+b \cos 2 \pi t)^{-1}, \text { where } 0<b<a ;
$$

so that (1) becomes the equation known from the problem of frequency modulation. In this case, (10) and (11) reduce to

$$
\mu=\left(a^{2}-b^{2}\right)^{-1 / 2} \text { and } M=(a-b)^{-1},
$$

and so the above inequalities supply explicit conditions for pairs $(a, b)$ which are sure to be of stable type. Needless to say, the resulting inequalities for $a$ and $b$ are just sufficient for stability. Incidentally, since $f(t)$ is now positive, Liapounoff's criterion, $\mu<4$, also is applicable.

\title{
LOWER BUCKLING LOAD IN THE NON-LINEAR BUCKLING THEORY FOR THIN SHELLS*
}

\section{By HSUE-SHEN TSIEN (Massachusetts Institute of Technology)}

For thin shells the relation between the load $P$ and the deflection $\epsilon$ beyond the classical buckling, load is very of ten non-linear. For instance, when a uniform thin circular cylinder is loaded in the axial direction, the load $P$ when plotted against the end-shortening $\epsilon$ has the characteristic shown in Fig. 1. If the strain energy $S$ and the total potential $\varphi=S-P \epsilon$ are calculated, their behavior can be represented by the curves shown in Figs. 2 and 3. It can be demonstrated that the branches $O C$ and $A B$ corresponds to stable equilibrium configurations and the branch $B C$ to unstable equilibrium configurations. The point $B$ is then the point of transition from stable to unstable equilibrium configurations.

It was proposed by the author in a previous paper ${ }^{1}$ that the point $A$ was the critical point for buckling of the structure under external disturbances, using the $S, \epsilon$ curve for "testing machine" loading and the $\varphi, P$ curve for "deadweight" loading. The load $P$ for the unbuckled configuration of the shell corresponding to the point $A$ was called

* Received April 2, 1947.

${ }^{1} \mathrm{H}$. S. Tsien, $A$ theory for the buckling of thin shells, J. Aero. Sciences 9, 373-384 (1942). 
the lower buckling load of the shell. The energy represented by the vertical distance from the point $A$ to the curve $B C$ is then the minimum external excitation required to cause the buckling at point $\dot{A}$.

However, if the external excitation is large, there is no reason why buckling cannot occur at the point $B^{\prime}$ directly under the point $B$. The minimum external excitation required is then given by the energy represented by the distance $B^{\prime} B$. This amount of energy is actually absorbed by the structure during buckling. Since the curve $B A$ represents the final state of the structure after buckling, for buckling to happen between $B^{\prime}$ and $A$, energy is absorbed, and for buckling to happen between $A$ and $C$, energy is released. But in any event, the lower limit of buckling load is definitely

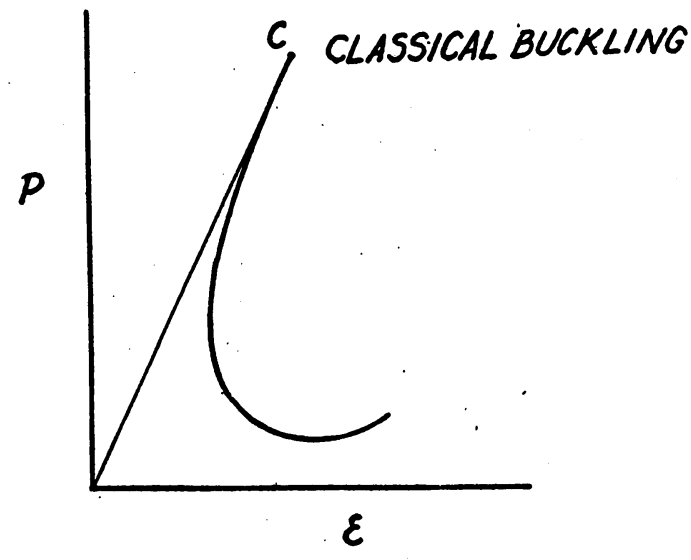

Fig. 1.

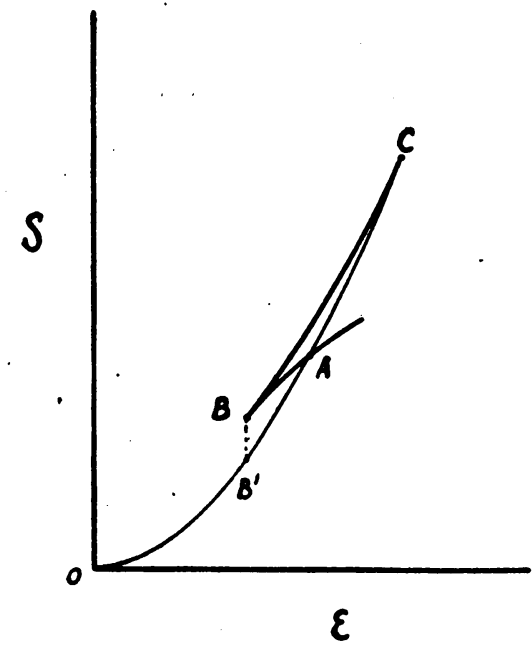

FIG. 2.

given by the point $B^{\prime}$, not the point $A$. Therefore the lower buckling load should be the load $P$ corresponding to the point $B^{\prime}$.

By referring to Figs. 11 and 13 of the aforementioned paper, and assuming a square' wave pattern, we find the lower buckling stress $\sigma$ of thin uniform cylindrical shells under axial load to be given by

$$
\sigma=0.42 E t / R
$$

for testing machine loading and

$$
\sigma=0.19 E t / R
$$

for deadweight loading. The corresponding values under the previously proposed criteria are $\sigma=0.46 E t / R$ and $\sigma=0.298 E t / R$ for the two cases.

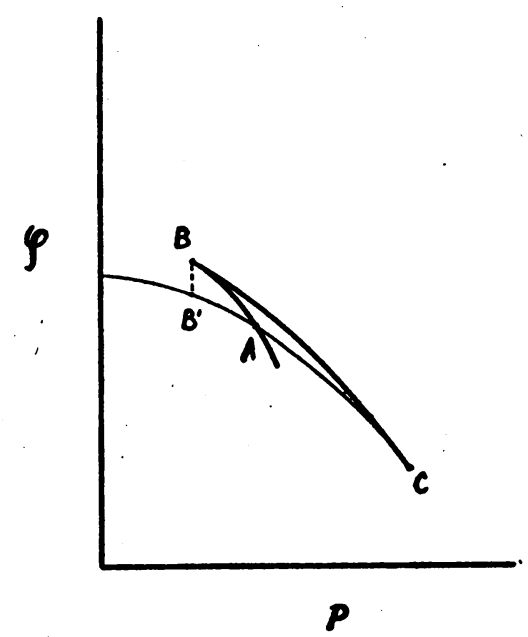

Fig. 3. 